Hier steht eine Anzeige.

\title{
Springer
}


Onkologe 2016 22:2-4

DOI 10.1007/s00761-015-2992-3

Online publiziert: 6. Januar 2016

๑) Springer-Verlag Berlin Heidelberg 2016

B.M. Ghadimi ${ }^{1}$ - C. Belka ${ }^{2}$ A. Neubauer ${ }^{3} \cdot$ K. Höffken ${ }^{4}$

${ }^{1}$ Klinik für Allgemein-, Viszeral- und Kinderchirurgie, Universitätsmedizin Göttingen, Georg-August-Universität, Göttingen

${ }^{2}$ Klinik und Poliklinik für Strahlentherapie und Radioonkologie, Klinikum der Ludwig-Maximilians-Universität München, München

${ }^{3}$ Klinik für Innere Medizin - Schwerpunkt Hämatologie, Onkologie und Immunologie, Universitätsklinikum Marburg, Marburg

${ }^{4}$ Universitätsklinikum Düsseldorf, Düsseldorf

\section{Molekulare Onkologie}

\section{Fundament der Präzisionstherapie maligner Erkrankungen}

Tumorigenese hat die Entwicklung der genomischen Medizin ermöglicht.

Für die „precision medicine“ absolut fundamental ist die molekulare Pathologie. $\mathrm{Zu}$ diesem Themenkomplex nehmen Kloth und Büttner Stellung und zeigen die Möglichkeiten der Prädiktion des Ansprechens, den Beitrag zur Prognose des klinischen Verlaufs, zur Durchführung von Surveillance-Strategien und in der allgemeinen Klassifikation von Tumorerkrankungen auf. Weiterhin wird die zunehmende Bedeutung der Detektion hereditärer Tumorprädispositionen und damit assoziierter Präventionsprogramme diskutiert.

Ein wesentlicher Themenkomplex der molekularen Onkologie sind molekular basierte Therapiestrategien. Beispielhaft eruieren Hessmann et al. epigenetische Strategien in der Krebstherapie. Erste Arzneistoffe, die über epigenetische Mechanismen wirken, kamen in den letzten Jahren neu auf den Markt und sind aus der Biomedizin nicht mehr wegzudenken (z. B DNA-Methyltransferase-Hemmstoffe und Inhibitoren von Histondeacetylasen). Neue epigenetische Ansätze wie z. B. Inhibitoren von Histonmethyltransferasen oder onkogenen Chromatinkomplexen haben zwar bislang noch keine Zulassung erreicht, werden aber intensiv in präklinischen und klinischen Studien getestet.

Ein zweiter Bereich hochinnovativer Therapiestrategien ist die Immunonkolo- gie. Hierzu berichten Dietrich und Theobald über den aktuellen Stand der Tumorvakzination, Checkpointinhibition und T-Zell-Therapien. Während die Rolle der Tumorvakzination bis jetzt noch unklar ist, zeigen die klinischen Erfolge der Checkpointinhibitoren und T-Zell-Therapie-Ansätze, dass die therapeutische Manipulation des Immunsystems eine neue und bei einigen Tumorerkrankungen bereits heute erfolgreiche onkologische $\mathrm{Be}$ handlungsstrategie darstellt.

Die mit Abstand erfolgreichste und etablierteste Form molekularer Therapiestrategien stellt das sog. Targeting von Kinasen dar. Diese Ansätze werden im Artikel von Michels et al. bearbeitet. Es sind v. a. Kinasen, die durch kleine Kinaseinhibitoren oder - im Falle von Rezeptorkinasen - auch durch monoklonale Antikörper gehemmt werden können. Wenn auch mit diesen Substanzen tumorbiologische Phänomene wie $\mathrm{z}$. B. Therapieresistenz nicht komplett ausgeschaltet werden können, so führen sie dennoch zu signifikanten Therapieverbesserungen, deren wirtschaftlicher Preis allerdings sehr hoch ist.

Durch das Verständnis von molekularen Mechanismen der Tumorentstehung und Tumorproliferation ist das Fundament für eine zielgerichtete (personalisierte) Präzisionstherapie maligner Tumoren gelegt worden. Das darauf aufbauende Gebäude wird in Zukunft vielfältige 
Hier steht eine Anzeige.

\section{算 Springer}


moderne Therapieoptionen beinhalten, die zu einer Verbesserung der Ergebnisse bei Reduktion der therapiebedingten Morbidität führen werden. Mit der molekularen Onkologie ist ein neues Zeitalter der Krebsbehandlung eingeläutet worden.

Für die Schriftleiter

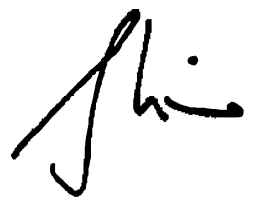

M. Ghadimi

Für die Herausgeber

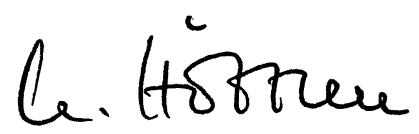

K. Höffken

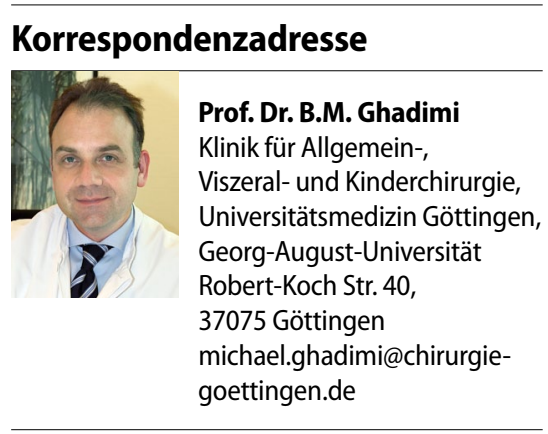

\section{Literatur}

1. Schwaederle M, Zhao M, Lee JJ et al (2015) Impact of precision medicine in diverse cancers: a metaanalysis of phase II clinical trials. J Clin Oncol 33(32):3817-3825

\section{ONKO-Internetportal}

Interdisziplinärer Austausch über

Krebstherapien von morgen

Bei der diesjährigen gemeinsamen Jahrestagung der Deutschen, Österreichischen und Schweizerischen Gesellschaften für Hämatologie und Medizinische Onkologie in Basel diskutierten rund 5500 Experten über die neuesten Ergebnisse aus der Grundlagenund der klinischen Forschung. Zudem standen zwei relativ neue, übergeordnete Forschungsgebiete im Zentrum des Austauschs: Wissenstransfer und Patientensicherheit. Vor Ort sprach das Redaktionsteam des ONKO-Internetportals mit Experten aus der klinischen Praxis. Im Fokus der VideoInterviews stehen die Betrachtung des breiten Spektrums neuer Therapieansätze sowie die Notwendigkeit einer interdisziplinären Versorgung von Patienten mit Krebserkrankungen. Erörtert werden unter anderem neue Substanzen und Kombinationen bei chronisch lymphatischer Leukämie sowie aktuelle Daten zum Nebenwirkungsprofil von neuen Arzneimitteln. Weitere Themen sind aktuelle Entwicklungen in der Hämatologie wie die PD-L1 Immuntherapie, Oberflächenmarker bei Lymphomen und neue Therapiestandards bei ZNS-Lymphomen. Darüber hinaus kommen die Methoden einer verfeinerten Diagnostik beim Multiplen Myelom zur Sprache. Alle Interviews und Berichte können ab sofort unter www.krebsgesellschaft.de/dgho2015 abgerufen werden.

Quelle: Deutsche Krebsgesellschaft e. V. (DKG) www.krebsgesellschaft.de

\section{Komplikationen nach Operationen am oberen Gastrointestinaltrakt}

Ein Großteil viszeralchirurgischer Operationen erfolgt am oberen Gastrointestinaltrakt, also an Ösophagus, Magen und Duodenum, wobei das Spektrum der Indikationen inzwischen hauptsächlich die Chirurgie von Malignomen umfasst. Dabei finden minimalinvasive Techniken sowie die laparoskopische und roboterassistierte Chirurgie zunehmend Anwendung.

Der Chirurg 11/2015 bietet Ihnen vier interessante Beiträge, die Einblicke in verschiedene Behandlungsformen und Management von Komplikationen ermöglichen:

- Management von Komplikationen bei endoskopischen Interventionen im oberen Gastrointestinaltrakt

- Management von Komplikationen unter und nach neoadjuvanter Therapie

- Management perioperativer Komplikationen nach Tumorresektionen im oberen Gastrointestinaltrakt

- Management von Spätkomplikationen nach Oesophagusresektion

Bestellen Sie diese Ausgabe zum Preis von 38,- EUR zzgl. Versandkosten bei Springer Customer Service Center Kundenservice Zeitschriften

Haberstr. 7

69126 Heidelberg

Tel.: +49 6221-345-4303

Fax: +49 6221-345-4229

E-Mail: leserservice@springer.com

Suchen Sie noch mehr zum Thema? Mit

e.Med, dem Online-Paket von Springer

Medizin, können Sie schnell und komfortabel

in über 500 medizinischen Fachzeitschriften recherchieren.

Weitere Infos unter springermedizin.de/eMed 